\title{
Exploring the effect of environmental thermal noise in the contra-lateral thermal asymmetry of the lower limbs
}

\author{
by E.I. Fuentes-Oliver*, C. García-Segundo*, R. Ortiz-Sosa*, R. Solalinde-Vargas ${ }^{* *}$ and R. Serrano-Loyola* \\ * Universidad Nacional Autónoma de México, Institute of Applied Sciences and Technology, Circuito Exterior \\ S/N, Ciudad Universitaria, Coyoacán, CDMX, Mexico, edgarfuentes@ciencias.unam.mx \\ ** Hospital General de México “Dr. Eduardo Liceaga”, Dr. Balmis 148, Doctores, Cuauhtémoc, CDMX, Mexico
}

\begin{abstract}
We study the environmental thermal noise effect on biomedical infrared imaging. The approach to discriminate noise from natural metabolic variability, rely on the initial reconsignment of contra-lateral thermal asymmetry between human lower limbs. Then we disambiguate this information from the environmental or background noise, by distinguishing its magnitude and spectral characteristics in the Fourier-space. From the spectral analysis one can unveil this kind of noise characteristics, remove it to correct the radiometric data and improve their true values.
\end{abstract}

\section{Introduction}

Infrared (IR) images display spatially the distribution of the heat radiated by an object [1]. Since ancient times [2], the infrared imaging (IRI) is particularly attractive in medical imaging. It is harmless and non-invasive in nature. Several studies, based on IR-images (IRIs), have explored how to extract macroscopic metabolic-related information on pathologies that cause changes in the thermal emission of the human skin [1,3].

Type 2 Diabetes Mellitus (DM2) is part of the so-called metabolic syndrome and is a worldwide health concern [4]. The diabetic foot disease (DFD), as DM2 co-morbidity, is a health and life risk for which aid from imaging methods is scarce. The DM2 prevalence is at rise in every country and thus, the DFD too. It has the most social, economic and health consequences, with devastating outcomes. The DFD is cause for lower limbs amputations, with down-effects on life-quality and dramatic shortening of the lifespan [4]. This etiology always includes neurological and vascular damage [4], leading to chronic alterations of the metabolic heat distribution. Under such conditions the lower extremities would display altered contra-lateral thermal asymmetry, respect of what could be the natural asymmetry. Details on the subject have been reported in [5].

Because the IRIs characteristics, we explore the intrinsic noise present in IRIs to understand how it could impact on the quality of the content of information. For the matter, we analyze the effect of local environment fluctuations, which are commonly neglected at time of analyzing IRIs. The general assessment is to assume the environment as stable under controlled room temperature, using domestic-like coolant systems [5]. For biomedical uses of IRI, such analysis is not good enough, and more accurate information is not available in a practical sense. With the aim to reduce measurement artifacts, initially we analyze the contra-lateral thermal asymmetry of the legs; then, for simplicity of making apparent the information features, we proceed to analyze the removal of thermal noise from IRIs in the Fourier-space.

\section{Methods}

We acquired a set of IRIs from a cohort of 36 clinically diagnosed patients with DM2 and 18 control volunteers, also diagnosed without the disease. Every subject was registered as participant in the supervised clinical protocol with strict informed-agreement and signed consent, HMG DI/10/301/4/115. HMG stand for Hospital General de México (from the Spanish language); the protocol is yet ongoing. The task is to achieve a large and reliable IRI data base.

The details on the methodology for the thermal asymmetry study are reported in [5]. In the current work we produce a comparative study, by getting information from a region of interest (ROI) of the IRIs. Initially we compute the contralateral thermal asymmetry without removing thermal noise, and then we repeat the process, removing then the thermal noise. At the end we compare the outcomes.

\subsection{Acquisition protocol}

The IRIs are acquired using an IR-camera (FLIR-A320) with a thermal sensitivity of $0.05 \mathrm{~K}$. The camera's userinterface allows adjustment of some acquisition parameters, such as: emissivity of the object, humidity, room temperature and the distance between the camera and the target. However, account only for general parametric conditions, interactive correction is not available. For the IRIs we report, we set in the camera for target emissivity of 0.95 , which we assume is the average emissivity of the human skin. We also set the humidity and room temperature, accordingly with the values monitored continuously, and that are updated for each acquisition set; i.e. for each patient.

The general guidelines used for IRIs acquisition, are near to these suggested in [6]. Additionally, we used a floor-mat for guiding standing-up postures. This is with the aim of standardize the postures and subject-to-camera distance, and thus the IRIs' views depicted on every image set. In the Fig. 1.a, we show a cartoon display of the mat. 
The colors are set it up to guide the individual's feet position. In Fig. 1.b, we show the relevant distances and physical configuration of the IRI setup. Before the acquisition, each subject was prepared with the ROI exposed to the environment, in rest condition, by 15 minutes. The aim of the waiting time is to reach thermal equilibrium between the subject and the environment. In the Figs. 1.c y 1.d, we display examples of the pictured images. These correspond to the anterior (front) and posterior (back) views of the legs.

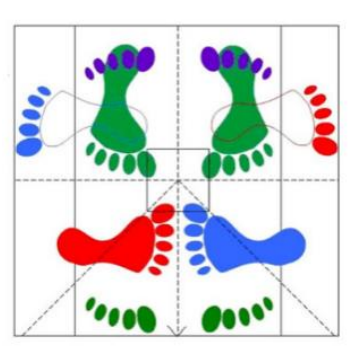

(a)

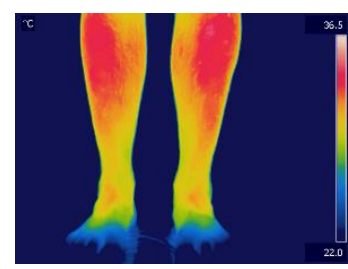

(c)

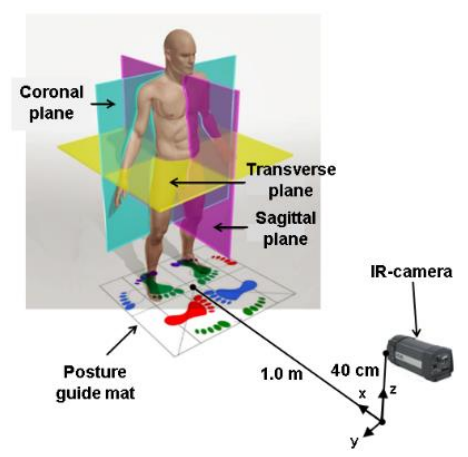

(b)

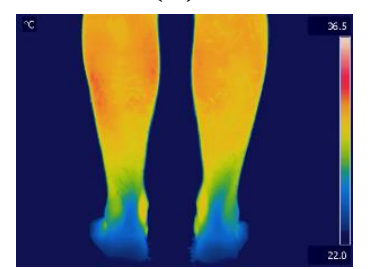

(d)

Fig. 1. (a) Posture guide mat. (b) Image acquisition setup. IR-images of (c) anterior and (d) posterior view of the legs.

\subsection{IR-images analysis}

For all the IRIs, we extract the radiometric information out of the selected ROI following the procedure graphically sketched in the Fig. 2. The region of interest for the study was the anatomical position of the tibial-fibular trunk and the birth of the dorsal artery of the foot. These regions are predominantly affected by peripheral vascular disease, and prone to neurological damage associated with the DFD.
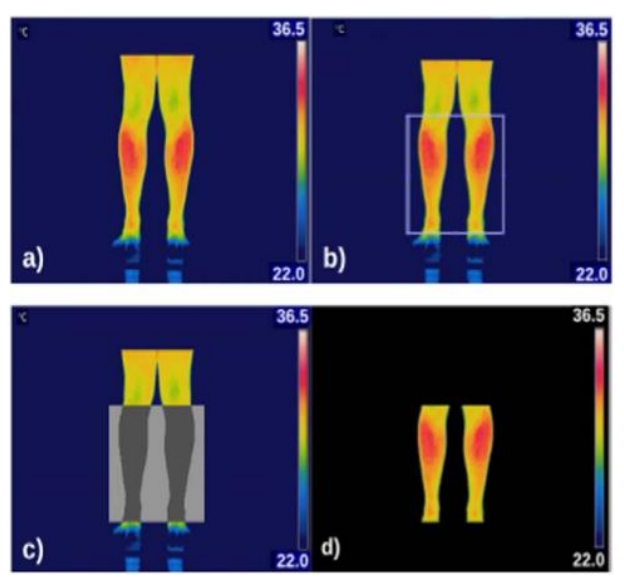

Fig. 2. Selection of the region of interest (ROI) in the IR-image. (a) Original image; (b) selection of the ROI; (c) highlighted ROI, dark gray region correspond to the legs and light gray zone is the background (environment); (d) segmented image.

\subsubsection{Removing thermal noise}

The environment thermal noise is calculated as the standard deviation of the temperature values of the background in each IR-image. We took care that no thermal information belonging to the portion of imaged legs, is 
misplaced on the chosen ROI. Each background image point is a $T_{B G_{k}}$ value. I.e. this vector represents the temperature values of the background (environment). We use this vector to get a value for the thermal noise $T_{\text {Noise }}$, defined as,

$$
T_{\text {Noise }}=\sqrt{\frac{1}{N} \sum_{k=1}^{N}\left(T_{B G_{k}}-\bar{T}_{B G}\right)^{2}} .
$$

Here, $N$ is the total number of k-temperatures, or vector components, in the ROI for background; $\bar{T}_{B G}$ is the average of these values. As defined above, $T_{\text {Noise }}$ is interpreted as the thermal fluctuations that affect the thermal information pictured on the IRIs. This value is directly subtracted from each temperature value of both the right leg $\left(T_{R_{i}+B G}\right)$ and the left one $\left(T_{L_{j}+B G}\right)$. These temperature values, $T_{R_{i}+B G}$ and $T_{L_{j}+B G}$, account for the thermal information before the noise removal. The thermal noise removal procedure is as follows:

$$
\begin{aligned}
& T_{R_{i}}=T_{R_{i}+B G}-T_{\text {Noise }} \\
& T_{L_{j}}=T_{L_{j}+B G}-T_{\text {Noise }} ;
\end{aligned}
$$

$T_{R_{i}}$ and $T_{L_{i}}$ represent the temperature values of each limb, right and left respectively, after subtracting $T_{\text {Noise }}$.

To look for differences between the thermal information of the legs before and after removing the noise, we Fourier-transform the histograms made with the temperature values of each limb. The subtraction between the Fourier transform of the thermal information with and without the environmental noise was obtained to show the existing differences.

\subsubsection{Computing the contra-lateral thermal asymmetry}

The next step is to get the relative thermal asymmetry between the lower limbs. For this purpose, we use the procedure and indices introduced in [5]. In it each image pixel represents an actual temperature value. Therefore, each pixel is labelled as $T_{R_{i}+B G}$ and $T_{L_{j}+B G}$, for the right and left legs, respectively. With these values and following the procedure we describe graphically in Fig. 3 the $\mathbf{R}$ and $\mathbf{L}$ vectors are built. These represent temperature vectors for the right and left legs, respectively. In addition, and from these vectors, we construct corresponding histograms of temperature.

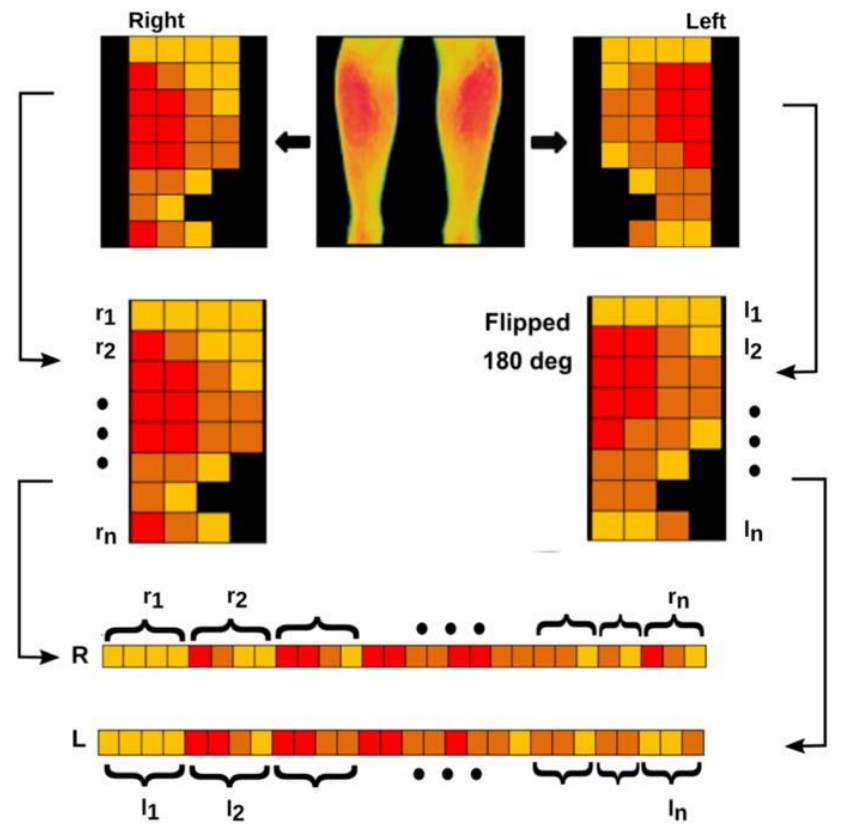

Fig. 3. Schematic representation of the process to construct the temperature vectors. Each leg is represented as a matrix of temperature values (upper image). To match the orientation of right leg, left leg is mirror-flipped 180 degrees (middle figure). The matrices' rows $\left(r_{j}\right.$ and $l_{j}$ ) are sequentially placed, one after the other, to create the $\boldsymbol{R}$ and $\boldsymbol{L}$ vectors.

The legs' relative thermal asymmetry is statistically estimated using the non-parametric Spearman's correlation $\left(\rho_{S}\right)$; between $\mathbf{R}$ and $\mathbf{L}$. Whilst the associated histograms are cross-correlated, to validate the extent of average similarity between $\mathbf{R}$ and $\mathbf{L}$; that is made relative to the temperature distributions and the associated frequencies; i.e. the number of times of occurrence of a k-temperature. 
From these correlations, we obtain the following indices, from which we establish the rate of asymmetry between the right and left legs.

Thermal asymmetry index (TAl): it represents the degree of asymmetry between the temperature vectors of each extremity, given as:

$$
T A I=\frac{1}{2}\left(1-\rho_{S}\right)
$$

$\rho_{S}$ is the Spearman's correlation coefficient.

Spectral asymmetry index (SAI): it stands for the degree of asymmetry between the temperature histograms associated with the temperature vectors of each extremity, given as:

$$
S A I=\frac{1}{2}\left(1-\rho_{X Y}\right)
$$

$\rho_{X Y}$ is the cross-correlation coefficient.

Total asymmetry index (TtAl): this is the magnitude of the vector resulting from assuming the corresponding TAI and $S A /$ pair as the respective coordinates.

$$
T t A I=\|(T A I, S A I)\|=\sqrt{T A I^{2}+S A I^{2}}
$$

We apply this procedure to the IRIs before and after removing the thermal noise, $T_{\text {Noise }}$, and we compare similitudes and differences. This was done for both groups, patients with DM and control volunteers without the disease, and for both acquisition views, front and back. Results of this analysis are shown following.

\section{Results and discussion}

The changes of the thermal information from the IRIs in the Fourier-space before and after the environmental noise removal are displayed in Fig. 4. In this figure we only show the changes in the left leg for the front and back views, and for both groups, control volunteers and patients. The results for the right leg are close similar.
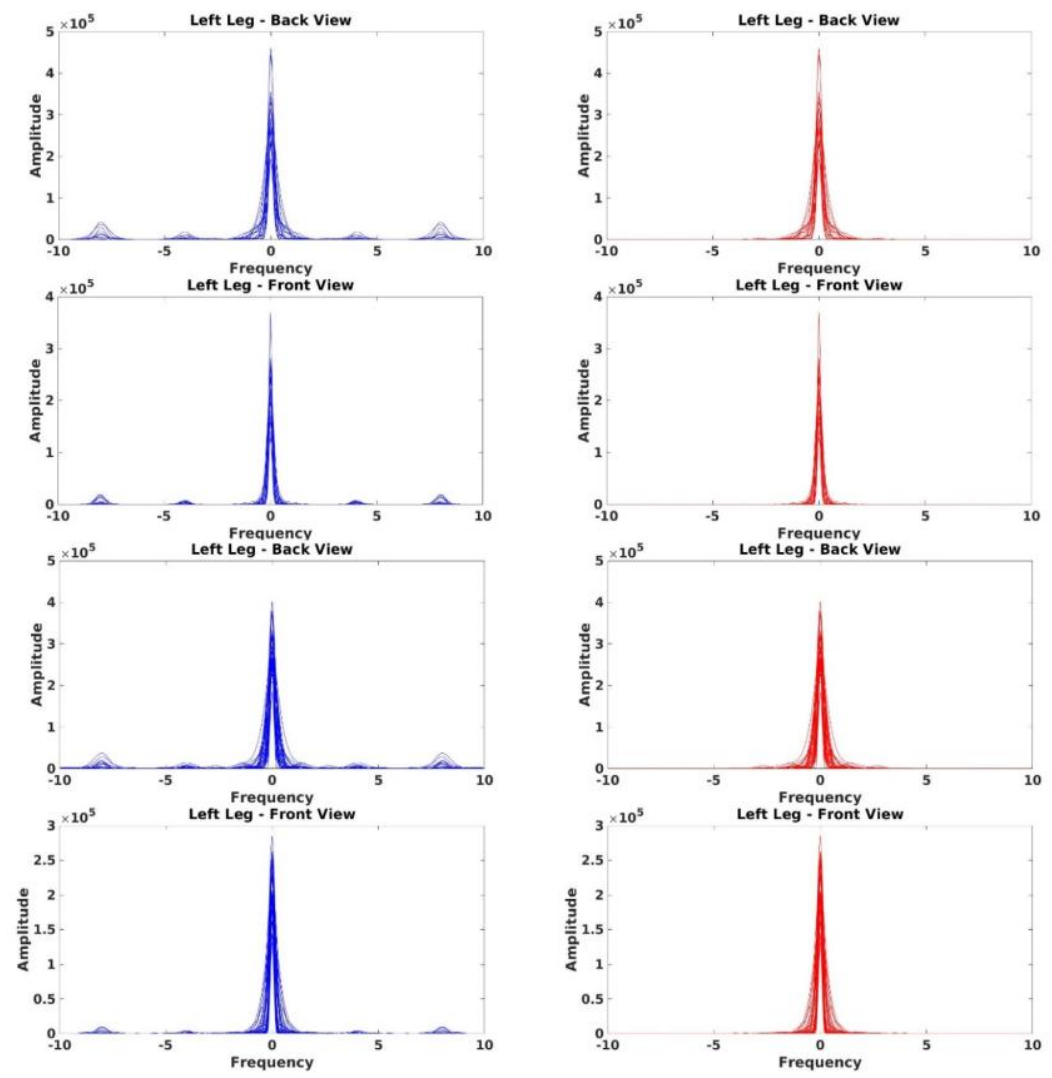

Fig. 4. Fourier transform of the thermal information with (left column) and without (right column) the thermal noise of the environment, for the left leg of control volunteers (four upper figures) and patients with DM (four lower figures). Figures in odd rows correspond to back view and that ones in even rows are for the front view. 


\section{$15^{\text {th }}$ Quantitative InfraRed Thermography Conference}

As can be seen in Fig. 4, when the thermal noise is not subtracted there are two lobes at each side of the central frequency. These lobes are high frequencies within which the noise interleave with actual image information. These lobes disappear when the thermal noise is removed. This suggest that removing the thermal noise in the way we proposed has the desired result.

The differences between the Fourier transform with and without noise are graphed in Fig. 5, this time only for the right leg. Results for the left leg are similar. As was noted before, the main differences are observed at higher frequencies, with a minimum effect in the central frequency.
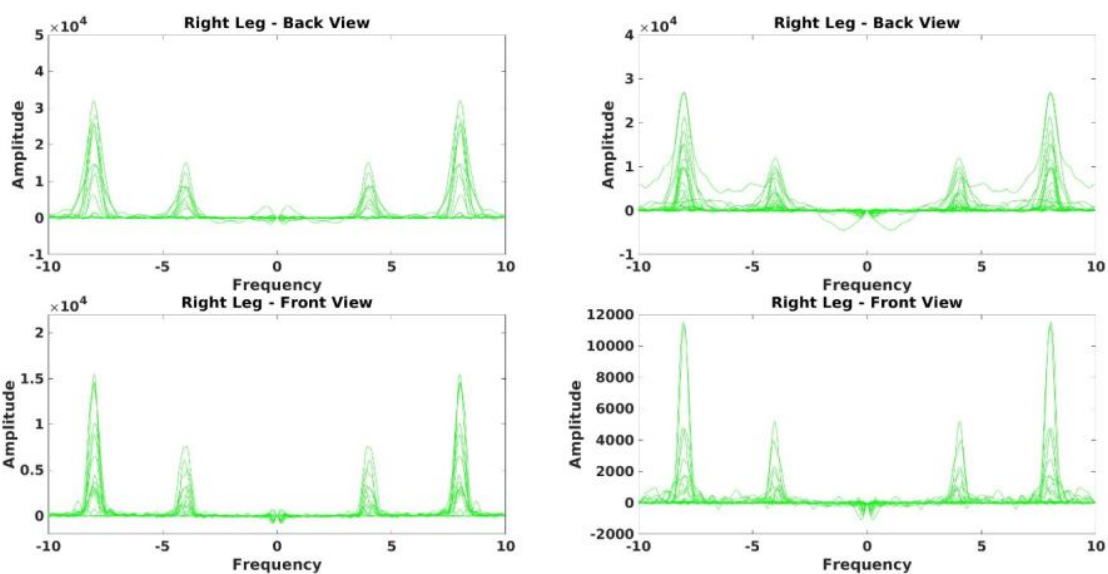

Fig. 5. Differences between the Fourier transform of the thermal information with and without the thermal noise of the environment, computed as the subtraction of the Fourier transform with noise minus the Fourier transform without noise. Left figures correspond to control volunteers and the right ones are for the patients with DM.

Since the effect in the central frequency as well as in the high frequencies could not be negligible in the thermal asymmetry, we explore the possible changes in the indices defined in Sec. 2.2, comparing these indices when are computed before and after removing the thermal noise (see Fig. 6).
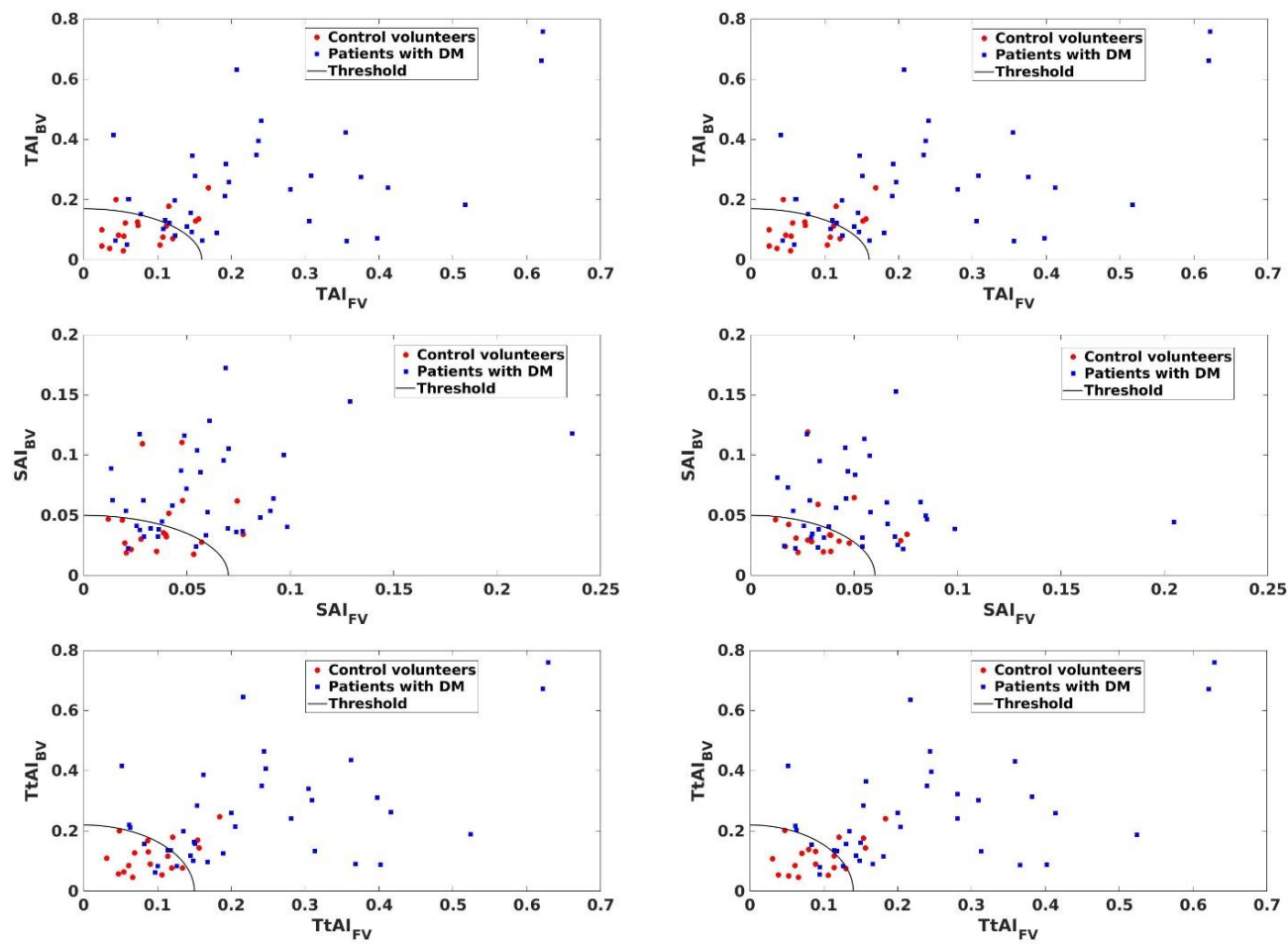

Fig. 6. Asymmetry indices computed with (left column) and without (right column) the thermal noise of the environment included in the thermal information. 
From Fig. 6, we note that the index TAI seems to have no change when the thermal noise is subtracted. Instead, the index SAI, and then the index TtAl, do show changes when the contribution of the environment is removed. These results are confirmed plotting the values of the subtraction between these indices before and after the thermal noise removal (see Fig. 7).

The former result is a consequence of the meaning of the Spearman's correlation coefficient, $\rho_{S}$, that is employed to compute the index TAI. $\rho_{S}$ only indicates if the tested variables are related for a monotonic function, and the trend of the data does not change after a subtraction, that only represents a translation of the data, not a change of the trend. On the other hand, the change of the index SAI is due its relationship with the temperature histogram, that is modified for each leg when the thermal noise is subtracted. As a direct consequence, the index TtAl is also modified (see Ec. (5)).
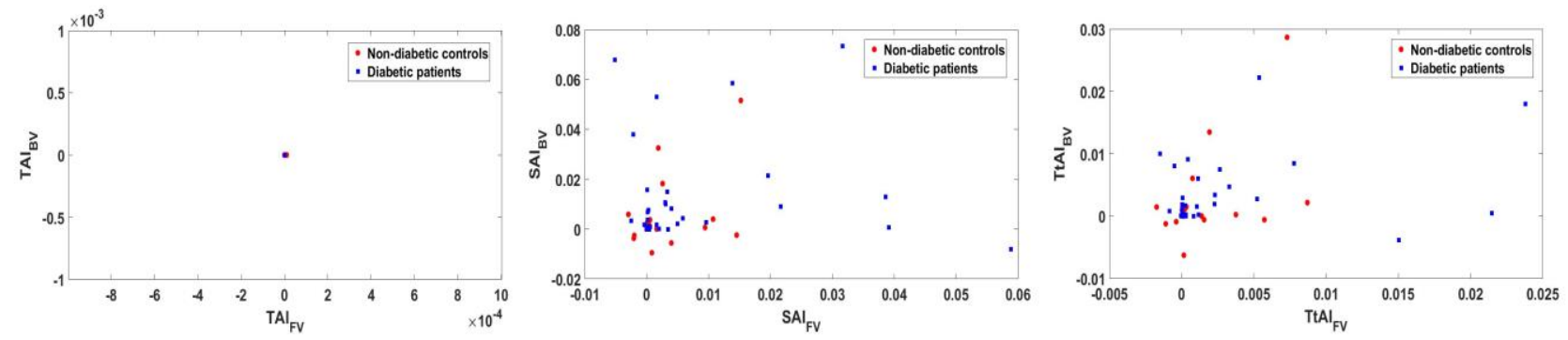

Fig. 7. Results of the subtraction between the asymmetry indices computed with and without the thermal noise of the environment included in the thermal information.

These results show that removing the environmental thermal noise has direct consequences over the contralateral thermal asymmetry. To quantify these changes, we compute the diagnostic performance of these indices through the ROC-analysis; then, the values of sensitivity and specificity are computed, considering the two studied groups.

According with the ROC-analysis, except for the TAI, that conserves its diagnostic performance (89\% of sensitivity and $72 \%$ of specificity) with and without noise, indices SAI and TtAI change their performances as follows: for the SAI the specificity increases from $67 \%$ to $72 \%$, and the sensitivity remains in $78 \%$; in the case of the TtAl the specificity remains in $78 \%$ and the sensitivity increases from $83 \%$ to $88 \%$ [5].

This improvement in the diagnostic performance of indices SAI and TtAl show that the thermal noise has nonnegligible effects in the evaluation of IRIs, changing the outcomes of the performed analysis.

\section{Conclusions}

The environmental thermal noise, pictured in the infrared images, is an inherent difficulty. From the current analysis, we disclose that there is a non-negligible effect of this noise in the evaluation of the infrared images. The influence of this thermal noise can be reduced by means of a subtraction of this environmental thermal noise from the thermal values of the region of interest in the Temperature-space.

The procedure described in this contribution leads to notable decrease in high frequencies, usually associated with noise in the Fourier-space. After this thermal noise removal, there is an improvement in the diagnostic performance of two of the three asymmetry indices evaluated in this work. Therefore, removing the environmental noise is a forceful task when quantitative true information is required. In biomedical applications it cannot be bypassed after the significative magnitude and type of artefacts caused by this noise.

The current approach is a preliminary evaluation of significance and magnitude. Further work is in progress to remove it from the infrared images and produce a cleaner analysis of contra-lateral thermal asymmetry of the legs.

\section{REFERENCES}

[1]. Grenn M. W. et al. Infrared camera and optics for medical applications. In Diakides M., Bronzino J. D. and Peterson D. R., Medical Infrared Imaging Principles and Practices. Boca Raton, FL: CRC Press 2013; 5:1-15.

[2]. Otsuka K. and Togawa T. Hippocratic thermography. Physiol. Meas. 1997; 18: 227-232.

[3]. Ring E. F. J. and Ammer K. Infrared thermal imaging in medicine. Physiol. Meas. 2012; 33: R33-R46.

[4]. World Health Organization. Global report on diabetes. 2016.

[5]. Fuentes-Oliver E. I. et al. Anomalous contra-lateral radiometric asymmetry in the diabetic patient. Biomed. Phys. Eng. Express 2019; 5: 065015.

[6]. Ring E. F. J., Ammer K. The technique of infrared imaging in medicine. Thermology International 2000; 10: 7-14.

[7]. Burgholzer P. and Günther H. Limits of Spatial Resolution for Thermography and Other Non-destructive Imaging Methods Based on Diffusion Waves. Int. J Thermophys. 2013; 34: 1617-1632. 